\title{
Proteomic profiling suggests a central role of STAT signaling during retinal degeneration in the $r d 10$ mouse model
}

\author{
Alice Ly ${ }^{1,2} \ddagger$, Juliane Merl-Pham ${ }^{1} \ddagger$, Markus Priller ${ }^{1}$, Fabian Gruhn ${ }^{1}$, Nicole Senninger ${ }^{1}$, Marius Ueffing ${ }^{1,3}$, Stefanie M. Hauck ${ }^{1 *}$ \\ ${ }^{1}$ Research Unit Protein Science, Helmholtz Zentrum München, German Research Center for Environmental Health (GmbH), München, Germany. \\ ${ }^{2}$ Research Unit Analytical Pathology, Helmholtz Zentrum München, German Research Center for Environmental Health (GmbH), Neuherberg, \\ Germany. \\ ${ }^{3}$ Centre of Ophthalmology, Institute for Ophthalmic Research, University of Tübingen, Tübingen, Germany.
}

\section{Content of supporting information:}

Supplemental Figure 1 Volcano plot of $r d 10$ vs wildtype fold changes and significance q-values at PN14 (A, blue), PN21 (B, red), and PN28 (C, green), and the combination of all three time points (D)

Supplemental table 1: Normalized abundances of proteins identified and quantified by LC-MS/MS, with corresponding ratios rd10/WT and significance values

Supplemental table 2: Significantly altered proteins at PN14 with q-value $<0.05$

Supplemental table 3: Significantly altered proteins at PN21 with q-value $<0.05$, with corresponding clusters in figure 2

Supplemental table 4: Significantly altered proteins at PN28 with q-value $<0.05$

Supplemental table 5: Primer sequences for qPCR

Supplemental table 6: Molecular functions overrepresented with clusters A and B with $\mathrm{p}<0.005$

Supplemental table 7: Molecular functions overrepresented with cluster E with $\mathrm{p}<0.005$ 
Supplemental Figure 1
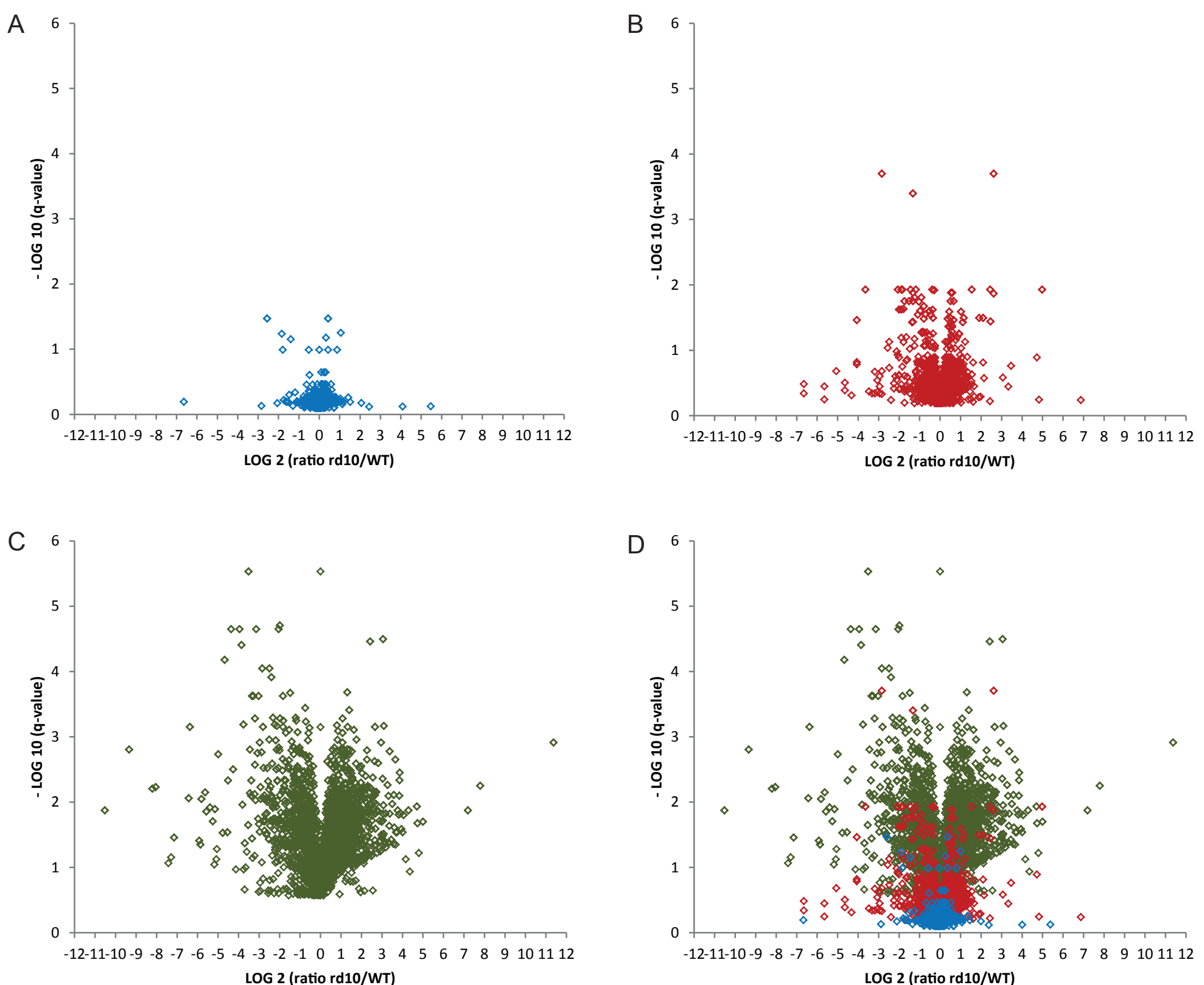